\title{
Ecología de murciélagos en el bosque seco tropical de Nariño (Colombia) y algunos comentarios sobre su conservación
}

\section{Bats ecology in the Dry Tropical Forest of Nariño (Colombia) and some commentaries about their conservation}

\section{Christian Cabrera-Ojedaa, ${ }^{1,2}$ Elkin A. Noguera-Urbano ${ }^{\star 1,2,3}$, Jhon Jairo Calderón-Leytón ${ }^{1,2}$ y Cristian Flórez Paí ${ }^{1,2}$}

1 Grupo de Ecología Evolutiva. Programa de Biología, Universidad de Nariño. Ciudadela Universitaria Torobajo.

2 Asociación GAICA. Pasto, Nariño, Colombia.

3 Grupo de Investigación de Biogeografía de la Conservación. Programa de Doctorado en Ciencias Biológicas. Departamento de Biología Evolutiva, Facultad de Ciencias, Universidad Nacional Autónoma de México, Apdo. 70-399. México, D. F.

${ }^{*}$ Autor para correspondencia:

Email Christian Cabrera-Ojeda: ccabrera516@gmail.com

Email Elkin A. Noguera-Urbano: elkalexno@gmail.com

Email Jhon Jairo Calderón-Leytón: jjcalderl@gmail.com

Email Cristian Flórez Paí: cristianfpai@gmail.com

\section{Resumen}

Los bosques secos tropicales son considerados como ecosistemas amenazados en Colombia, porque están localizados en áreas con actividades humanas intensivas. Sin embargo, los efectos de la pérdida de hábitat sobre la distribución y abundancia de murciélagos en esta región es poco conocida. En este estudio se analizó la riqueza de especies, composición y abundancia relativa de los murciélagos en tres tipos de vegetación con diferente grado de intervención en el Bosque Seco Tropical de Nariño (Mesi: Matorral espinoso seco intervenido, Mesc: Matorral espinoso seco conservado, Bsi: Bosque semideciduo intervenido), Colombia. Para ello se capturaron ejemplares con 10 redes de neblina durante ocho noches en cada tipo de vegetación, para un total de 24 noches. Se registraron nueve especies a partir de la captura de 60 ejemplares. EI Mesc (6 especies) presentó la mayor riqueza, mientras que Mesi presentó la menor riqueza de especies (4 especies), lo cual puede estar relacionado con el grado de perturbación de la zona. El índice de Sorensen $(0,33-0,38)$ indicó que las tres poblaciones podrían representar un solo ensamblaje. Las especies de mayor abundancia fueron Artibeus lituratus (Mesi), A. planirostris (Bsi) y Carollia perspicillata (Mesc), las cuales se han relacionado con vegetación que presentan algún grado de intervención o en regeneración. Criterios como la baja riqueza de especies, la dominancia de las tres especies mencionadas y la presencia de Desmodus rotundus sugieren que el grado de intervención es similar en los tres tipos de vegetación. Por lo tanto es necesario establecer programas de conservación de murciélagos y proyectos de restauración en el área.

Palabras claves: diversidad; desertificación; deterioro ambiental; ecología; riqueza.

\section{Abstract}

Tropical dry forests are considered as threatened ecosystems in Colombia, because they are located in areas with intensive human activities. However, the effects of habitat loss on the distribution and abundance of bats in this region are little known. The purpose of this study was to analyze species richness, composition and relative abundance of bats in three vegetation types with different degrees of intervention in the Tropical Dry Forest of Nariño (Mesi: Intervened dry thorn scrub, Mesc: Preserve dry thorn scrub, Bsi: Intervened semideciduous tropical forest), Colombia. We captured bats using 10 mist nets over 24 nights (eight nights in each vegetation type). Nine species were recorded from the capture of 60 specimens. The Mesc had the higher richness (6 species), while the Mesi had the lower richness (4 species). This is probably related with the high degree of human disturbance in the area. Sorensen index $(0.33-0.38)$ indicated that the three populations represent the same community. A. lituratus (Dtsi), A. planirostris (Tsfi) and C. perspicillata (Dtsp) showed the lower abundance, this condition has been associated with forest in process of regeneration or forest with human intervention. Although the best preserved area is the Mesc, criteria as the low species richness, dominance of the three bat species and the presence of Desmodus rotundus suggest that the degree of human intervention is similar in the three types of vegetation. Therefore it is necessary to establish bat conservation programs and restoration projects in the area.

Keywords: desertification; diversity; ecology; environmental degradation; richness.

Citación:

Cabrera-Ojeda C., E.A. Noguera-Urbano, J.J. Calderón-Leytón y C. Flórez Paí. 2016. Ecología de murciélagos en el bosque seco tropica de Nariño (Colombia) y algunos comentarios sobre su conservación. Revista peruana de biología 23(1): 027 - 034 (Abril 2016). doi: http:// dx.doi.org/10.15381/rpb.v23i1.11830

$\begin{array}{ll}\text { Presentado: } & 05 / 03 / 2015 \\ \text { Aceptado: } & 08 / 03 / 2016\end{array}$

Publicado online: $28 / 05 / 2016$

\section{Información sobre los autores:}

CC-O diseñó el estudio, obtuvo los datos de campo, realizó los análisis y la interpretación de datos. EAN-U diseño el estudio, realizó los análisis y la interpretación de datos, se encargó de la redacción del artículo. JC-L y CF lideraron el proyecto, realizaron revisiones críticas del manuscrito. Los autores no incurren en conflictos de intereses.

Fuentes de financiamiento: El presente trabajo se realizó gracias a financiamiento de: The Nature Conservancy (TNC), el Fondo Conserva Colombia y la Asociación GAICA, Pasto Nariño.

Journal home page: http://revistasinvestigacion.unmsm.edu.pe/index.php/rpb/index

(c) Los autores. Este artículo es publicado por la Revista Peruana de Biología de la Facultad de Ciencias Biológicas, Universidad Nacional Mayor de San Marcos. Este es un artículo de acceso abierto, distribuido bajo los términos de la Licencia Creative Commons Atribución-NoComercial-Compartirlgual 4.0 Internacional.(http://creativecommons.org/licenses/by-nc-sa/4.0/), que permite el uso no comercial, distribución y reproducción en cualquier medio, siempre que la obra original sea debidamente citadas. Para uso comercial, por favor póngase en contacto con editor.revperubiol@gmail.com. 


\section{Introducción}

Los bosques secos tropicales (Bs-T) son una formación intermedia entre la sabana tropical y el bosque húmedo tropical (Sánchez et al. 2007), que presenta uno o dos periodos largos de deficiencia de agua (IAvH 1998). En el departamento de Nariño, suroccidente de Colombia, el Bs-T se encuentra entre montañas del Macizo Colombiano sobre las cuencas de los ríos Patía, Mayo y Juanambú, en límites con el departamento del Cauca (Delgado et al. 2007). El Bs-T en Nariño es un área prioritaria de investigación y posible conservación por su vulnerabilidad, las especies restringidas que lo habitan y el número de poblaciones humanas que dependen de sus recursos (Delgado et al. 2007). Además su ubicación interandina lo caracteriza como una de las formaciones secas de importancia para Colombia, por su aparente aislamiento histórico de otras zonas secas del país (Sánchez et al. 2007).

Los Bs-T han sufrido grandes transformaciones por prácticas como la agricultura, ganadería y deforestación, que han causado su reducción hasta remanentes de vegetación (IAvH 1998, Miles et al. 2006). Cuando el deterioro es avanzado, en los suelos del Bs-T se produce la desertificación, es decir aumento en la salinización de los suelos y el incremento de la erosión por el viento (García et al. 2014). En Colombia el $27 \%$ de las áreas que presentan desertificación se encuentran en el Bs-T (García et al. 2014). Por lo tanto la degradación de los suelos es uno de los principales problemas que enfrentan las poblaciones humanas y los animales silvestres que habitan el Bs-T.

Los murciélagos son de especial interés en el análisis de los cambios ambientales de los Bs-T. Se ha sugerido que los cambios en la diversidad y composición de las poblaciones de murciélagos están relacionados con la disponibilidad de fuentes de alimento y/o la conservación de sus ambientes (Chávez \& Ceballos 2001, Jiménez-Ortega \& Mantilla-Meluk 2008, Medellín et al. 2010). Esto se debe a la estrecha relación de los murciélagos con grupos particulares de plantas, por ejemplo en los bosques secos tropi- cales los nectarívoros intervienen en la polinización de algunas cactáceas (Ruíz et al. 1997) y otros con la dispersión de semillas (Sánchez et al. 2007).

Pese a reconocerse la persistente degradación de los Bs-T en Nariño (Delgado et al. 2007) y la importancia de los murciélagos para diagnosticar el estado de los ecosistemas (García-Morales et al. 2012), se sabe poco sobre la ecología de los murciélagos en la zona. Entre los estudios realizados en el Bs-T de Nariño se cuenta con un listado de 12 especies presentado por Alberíco \& Negret (1992), la descripción de una nueva especie de murciélago (Anuora carishina, Mantílla-Meluk \& Baker 2010) con localidad tipo en Taminángo (localidad ubicada en el Bs-T nariñense) y una lista de chequeo con nueve especies (Ramírez-Chaves \& Noguera-Urbano 2010). Tan solo el estudio de Sánchez et al. (2007) se describen atributos ecológicos de las poblaciones de murciélagos en los Bs-T de Nariño y se menciona la disminución de la abundancia y riqueza de los murciélagos por la presencia de actividades humanas.

El objetivo de este estudio fue analizar la riqueza, diversidad, composición y similitud del ensamblaje de murciélagos en tres tipos de vegetación con diferente grado de intervención en el Bs-T de Nariño. Considerando las especies registradas en este estudio se hacen comentarios sobre la conservación de los murciélagos y su entorno en el BS-T de Narińo. Se espera que los resultados de este estudio puedan ser empleados como línea base en el desarrollo de programas de conservación de la zona.

\section{Material y métodos}

Zona de estudio.- En el Bs-T de Narińo predominan pastizales que son resultado de la ganadería intensiva, sin embargo se observan parches de bosques a lo largo de quebradas y ríos. En la zona predominan fragmentos de formaciones vegetales de familias como Cactaceae, Capparidaceae y Zygophyllaceae. Los tres tipos de vegetación en donde se realizaron los muestreos son los siguientes (Fig. 1):

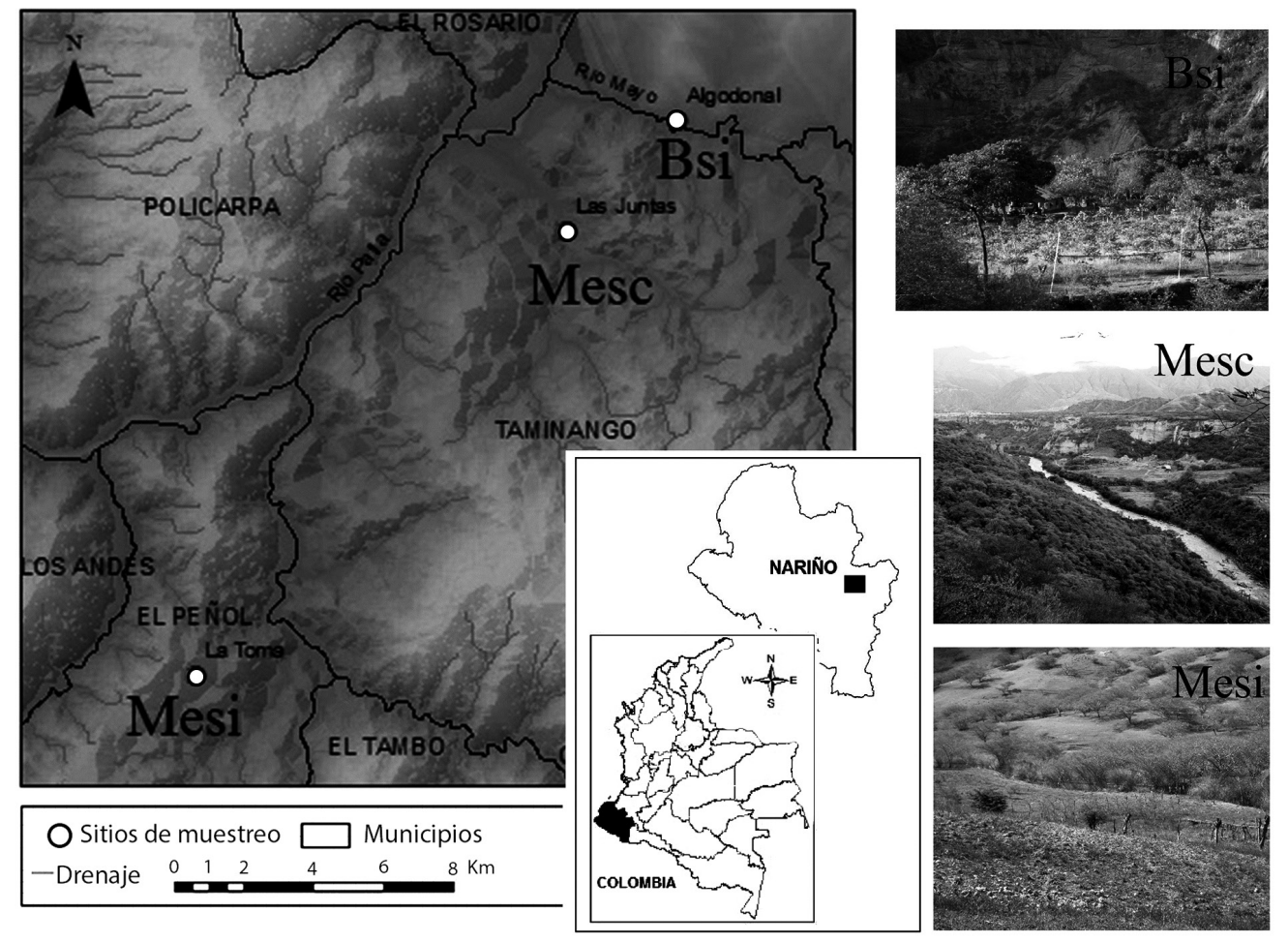

Figura 1. Ubicación de los sitios de muestreo en los ecosistemas secos del Departamento de Nariño. 
Matorral espinoso seco intervenido (Mesi).- La localidad se encuentra en el Municipio de El Peñol, Vereda La Toma (N $\left.1^{\circ} 31^{\prime} 46^{\prime \prime}-\mathrm{W} 77^{\circ} 25^{\prime} 14^{\prime \prime}, 655 \mathrm{~m}\right)$. Se caracteriza por vegetación semidensa, fragmentada, xerofítica, espinosa, achaparrada con presencia de cactus (Opuntia sp. y Pilocereus sp.) y plantas de látex (Croton sp. \& Euphorbia sp.) (Jurado y\& Herrera, 2014). Son frecuentes los cultivos de maracuyá. La vegetación natural se encuentra sobre las riberas de la quebrada Pindiguara, a pocos kilómetros del río Juanambú. En el sitio las actividades de ganadería provocan el pisoteo de plantas, fragmentación de la vegetación y contaminación de las escasas fuentes hídricas.

Bosque semideciduo intervenido (Bsi).- Localidad en el Municipio de Taminango, Vereda Algodonal (N 140'21"'W 77¹7'44", $580 \mathrm{~m}$ ). El lugar de muestro se encontraba a $300 \mathrm{~m}$ del río Mayo. Este tipo de bosque se caracteriza por la presencia mayoritaria de especies arbóreas y sotobosque con especies caducas durante la época seca del año, como es el caso de Handroanthus chrysanthus, Acacia pennatula y Cytharexyllum kunthianum. El sotobosque esta formado por arbustos de Zanthoxylum fagara, Croton ferrugineus y Jatropha gossypiifolia (Jurado \& Herrera, 2014). En algunos sitios se encuentran cultivos de papaya, mango, maní, sandía y limón. En el lugar se encuentran saraconchos o quebradas secas donde la vegetación tiene estratos arbóreo y arbustivo.

Matorral espinoso seco conservado (Mesc).- Se encuentra en el Municipio de Taminango, Vereda Las Juntas (138'38'"N $77^{\circ} 19^{\prime} 29^{\prime \prime} \mathrm{W}, 640 \mathrm{~m}$ ). Presenta mayor presencia de individuos de los estratos subarboreo y arbustivo que los otros dos lugares y en particular que en la vereda La Toma (El Peñol) (Jurado y Herrera, 2014). Los bosques se mezclan con cultivos de mandarina, maní y limón. La vegetación ribereña es escasa y ha sido transformada a cultivos. Se encuentra ganado bovino, caprino y porcino. En la zona se encuentra un área de conservación privada de aproximadamente $200 \mathrm{Ha}$ (Obs. pers. CC-O, CF).

Captura de murciélagos.- Durante 24 días entre los meses de octubre y noviembre del 2014 (inicio época de lluvia), se realizaron muestreos en las tres localidades caracterizadas como Bosques Secos Tropicales de Nariño. En cada localidad se utilizaron 10 redes de neblina $(2.5 \mathrm{~m}$ x $10 \mathrm{~m}$, ojo de malla $16 \mathrm{~mm}$ ) separadas en dos puntos de muestreo cubriendo vegetación natural, cultivos o cerca a fuentes de agua. Se ubicaron cinco redes en cada punto de muestreo, a distancia aproximadas entre puntos de 500 y $2000 \mathrm{~m}$. La apertura de redes en cada localidad fue de ocho noches consecutivas, con técnicas en transectos en subdosel (Tirira 1998), con una intensidad de muestreo de 5 horas y periodos de revisión de 30 minutos. Las redes se cambiaron de lugar cada tres días tratando de abarcar los distintos tipos de uso del suelo. Además, se visitaron cuevas y sitios de refugio donde se hizo captura manual de algunos ejemplares.

Para cada ejemplar capturado se registró la fecha y hora de captura, especie, sexo, condición reproductiva y morfometría básica. Aquellos especímenes de difícil determinación taxonómica fueron fijados en piel seca-cráneo extraído, esqueleto o en fluidos (Simmons \& Voss 2007). La identificación de los ejemplares colectados fue realizada en el Museo de la Universidad de Nariño (MUN-CZ), con literatura especializada (Gardner 2007) y algunas revisiones de géneros.
Análisis de información.- Se estimó la riqueza por cobertura de muestreo, que es la proporción del número total de individuos en una comunidad que pertenecen a la especie presente en la muestra (Chao \& Jost 2012). Además se describió la abundancia relativa y composición del ensamblaje de murciélagos. Se comparó la riqueza entre sitios obteniendo la cobertura de muestreo (Chao \& Jost 2012), en el programa iNEXT (http:// chao.stat.nthu.edu.tw/blog/software-download/) (Hsieh et al. 2015). La cobertura de muestreo es una medida de la completitud de la muestra con respecto al tamaño de la comunidad, así una comunidad bien muestreada debería tener una cobertura de 100\% (completamente muestreada; Chao \& Jost 2012). La riqueza total por localidades fue estimada calculando curvas de acumulación de especies (días como unidades de muestreo y 100 aleatorizaciones) a partir de tres estimadores: uno no paramétrico (Chao 1), otro basado en abundancia (ACE) y un estimador paramétrico (Michaelis-Menten) (Gotelli \& Colwell 2011) en el programa EstimateS v.9.0 (Colwell 2013).

La diversidad fue medida en términos del número efectivo de especies, que expresa la diversidad que tendría un ensamblaje con un número de especies igualmente abundantes (Jost 2006, Moreno 2011). Se estimaron dos tipos de diversidad, la diversidad alfa y la beta. Para describir la composición de especies de murciélagos en las tres localidades se graficaron curvas de rango-abundancia de Whittaker $\left(\log _{10}\right.$ de la abundancia sobre eje Y; Magurran 2004).

La diversidad alfa en cada localidad se midió teniendo en cuenta la propuesta de Jost (2006), que se basa en los números de Hill, donde la diversidad se expresa como ${ }^{q} \mathrm{D}$ y $q$ puede tomar los valores de " 0 " (diversidad expresada como riqueza observada), "1" (especies abundantes y raras tienen igual importancia, equivale al exponencial del índice clásico de Shannon) o "2" (mayor importancia de las especies abundantes, equivale al inverso del índice de Simpson). Este método tiene más sentido biológico que los índices clásicos de diversidad porque el índice se expresa en número efectivo de especies, y no en unidades de información que son complejas de interpretar (Jost 2006).

La diversidad alfa entre los sitios se graficó junto a los intervalos de confianza al 95\%. Los análisis estadísticos se llevaron a cabo en el programa PAST v. 2.17 (Hammer 2001). La diversidad beta se calculó entre pares de sitios a través del índice de Sorensen (Baselga 2010).

\section{Resultados}

Entre los tres tipos de vegetación se capturó un total de 60 murciélagos de nueve especies (Tabla 1), pertenecientes a la familia Phyllostomidae. La subfamilia Phyllostominae presentó tres especies, mientras que Stenodermatinae y Glossophaginae presentaron dos especies cada una. Las subfamilias Carollinae y Desmodontinae solo presentaron una especie. La riqueza entre localidades fue heterogénea, seis especies caracterizaron al Mesc como el sitio de mayor riqueza, los otros dos tipos de vegetación tuvieron cinco y cuatro especies (Tabla 1). El sitio con la mayor cobertura de muestreo fue Mesi (95.8\%), mientras que el Bsi (75\%) y el Mesc ( $92 \%)$ tuvieron una menor cobertura (Tabla 2 ). Los valores representan el porcentaje de especies considerando el estimador bajo el esfuerzo de muestreo alcanzado.

El número de especies de los tres sitios difiere tan solo por una o dos especies entre sí (Tabla 1), sin embargo se obtuvieron 
Tabla 1. Murciélagos registrados durante el muestreo en el Matorral espinoso seco intervenido (Mesi), el Bosque semideciduo intervenido (Bsi) y el Matorral espinoso seco conservado (Mesc).

\begin{tabular}{|c|c|c|c|c|c|}
\hline Familia & Subfamilia & Especie & Mesi & Bis & Mesc \\
\hline \multirow{9}{*}{ 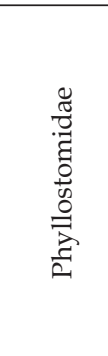 } & Carollinae & Carollia perspicillata & $x$ & & $x$ \\
\hline & Desmodontinae & Desmodus rotundus & $x$ & $x$ & $x$ \\
\hline & \multirow{2}{*}{ Glossophaginae } & Glossophaga soricina & & $x$ & $x$ \\
\hline & & Anoura peruana & $x$ & & \\
\hline & \multirow{3}{*}{ Phyllostominae } & Phyllostomus discolor & & & $x$ \\
\hline & & Vampyrum spectrum & & $x$ & \\
\hline & & Micronycteris minuta & & $x$ & \\
\hline & \multirow{2}{*}{ Stenodermatinae } & Artibeus lituratus & $x$ & & $x$ \\
\hline & & Artibeus planirostris & & $x$ & $x$ \\
\hline \multicolumn{3}{|c|}{ Total de especies por sitio } & 4 & 5 & 6 \\
\hline
\end{tabular}

marcadas diferencias en la abundancia (Tabla 2). El número de murciélagos capturados en el Mesi y el Mesc duplican la abundancia de Bsi (Tabla 2). La riqueza de especies presentan una completitud de muestreo entre el $36.2 \%$ y el $100 \%$ para el Mesi y su riqueza observada se ajustó a la predicción del estimador Chao 1 (Fig. 2a). En el Bsi se obtuvo una completitud entre el $43 \%$ y el $64.5 \%$ (Fig. 2b), mientras que para el Mesc tuvo un intervalo entre el $22.7 \%$ y el $92.6 \%$ (Fig. 2c).

Con respecto a la diversidad en base a $q=1$ no se observan diferencias significativas entre los tres tipos de vegetación (Tabla 3, Fig. 3b). El Bsi fue el sitio más diverso con 3.7 especies efectivas. El Mesi presentó una diversidad expresada como 2.9 especies efectivas, los intervalos de confianza son bastante amplios debido a las bajas abundancias. La diversidad total de murciélagos presenta valores de 5.27 y 3.95 especies efectivas para $q=1$ y $q=2$ respectivamente (Tabla 3 ).

La estructura del ensamblaje de murciélagos basada en las curvas de rango-abundancia (Fig. 4) indicó que hay diferencias en la dominancia de especies en los tres sitios. Entre el Mesi y el Mesc hubo un recambio de especies dominantes, en el primero la especie dominante fue Artibeus lituratus (Fig. 5a), seguida por Carollia perspicillata. Mientras que en el Mesc C. perspicillata fue la especie dominante, seguida por $A$. lituratus y Phyllostomus discolor (Fig. 4). En el Bsi la especie dominante fue A. planirostris,

Tabla 3. Valores de diversidad en orden de $q$ para el conjunto de los tres tipos de vegetación.

\begin{tabular}{ccc}
\hline Valor de $q$ & Diversidad & $\begin{array}{c}\text { Desviación } \\
\text { estándar }\end{array}$ \\
\hline 0 & 9.0000 & 3.0000 \\
1 & 5.2748 & 1.4977 \\
2 & 3.9560 & 1.1303 \\
\hline
\end{tabular}

Tabla 2. Cobertura de muestreo para el Matorral espinoso seco intervenido (Mesi), el Bosque semideciduo intervenido (Bsi) y el Matorral espinoso seco conservado (Mesc). Se indica el número de individuos (N), la riqueza observada (S obs), el resultado del estimador Chao 1 y el porcentaje de cobertura de muestreo (C. Muestreo) para cada sitio.

\begin{tabular}{lcccc}
\hline Sitio & $\mathrm{N}$ & S obs & Chao 1 & C. Muestreo \\
\hline Mesi & 22 & 4 & 4.48 & $95.85 \%$ \\
Besi & 12 & 5 & 7.75 & $75 \%$ \\
Mesc & 26 & 6 & 7.92 & $92.6 \%$ \\
\hline
\end{tabular}

seguida por Desmodus rotundus. Otras especies como Micronycteris minuta, Vampyrum spectrum (Fig. 5b) y Glossophaga soricina presentaron tan solo un individuo capturado sin ser registrados en los tres sitios de muestreo. La diversidad beta estimada a partir del índice de disimilaridad de Sorensen varió entre $0.33-0.38$ indicando que posiblemente las tres poblaciones podrían representar una mismo ensamblaje (Fig. 6). Se resalta la presencia de $V$. spectrum en los Bs-T de Nariño. Una especie endémica del neotrópico y considerada casi amenazada (NT) por presentar poblaciones aisladas y pequeñas y la disminución de su hábitat natural (Aguirre et al. 2008).

\section{Discusión}

Los Bs-T de Nariño se encuentran ubicados dentro de la cuenca del rio Patía, de acuerdo a algunos autores (Alberico \&
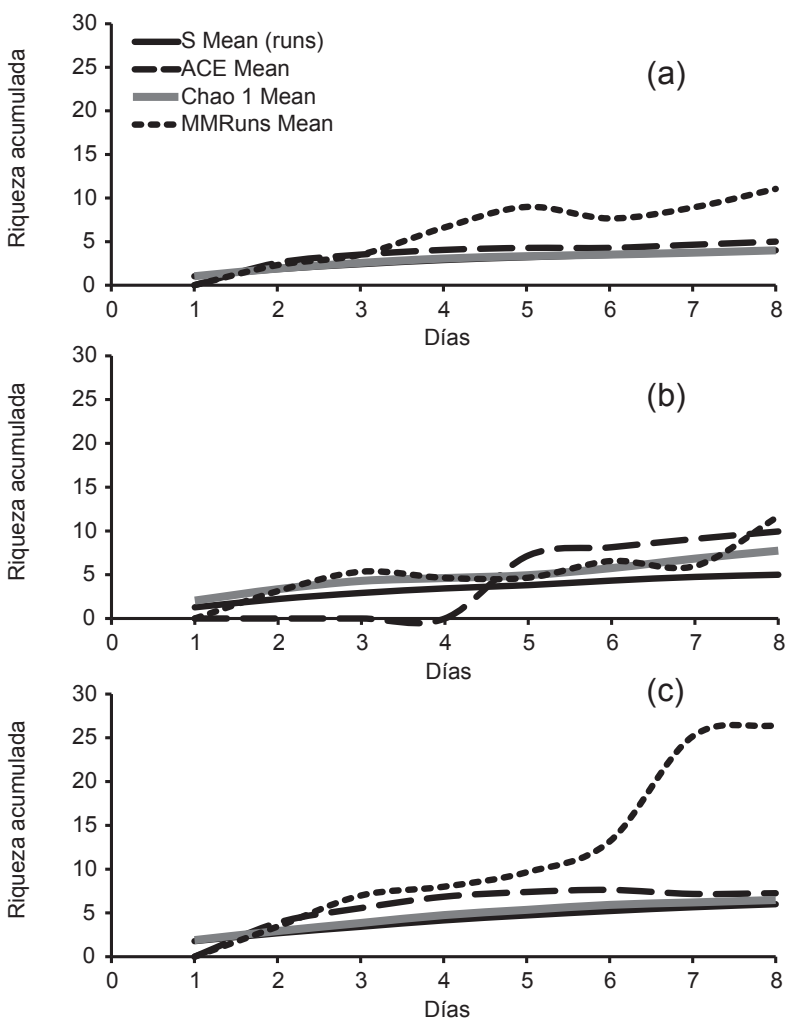

Figura 2. Curvas de acumulación de especies para los tres tipos de bosque analizados. a. Matorral espinoso seco intervenido (Mesi), b. Bosque semideciduo intervenido (Bsi) y c. Matorral espinoso seco conservado (Mesc). 

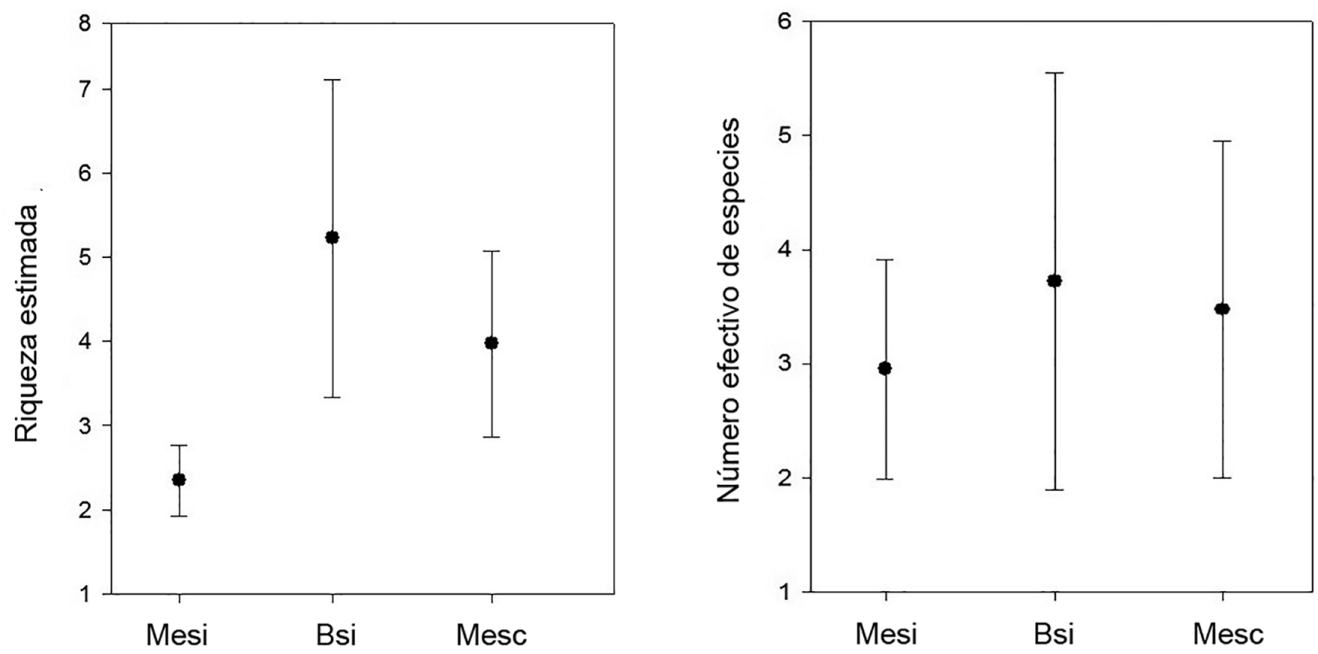

Figura 3. Valores de riqueza de especies. a. Comparada a un $79 \%$ de cobertura de muestreo y b. Diversidad base $q=1$ de los tres sitios. Los puntos $(\bullet)$ indican las medias y las líneas horizontales en cada intervalo representan los valores máximos y mínimos con una significancia del $95 \%$.

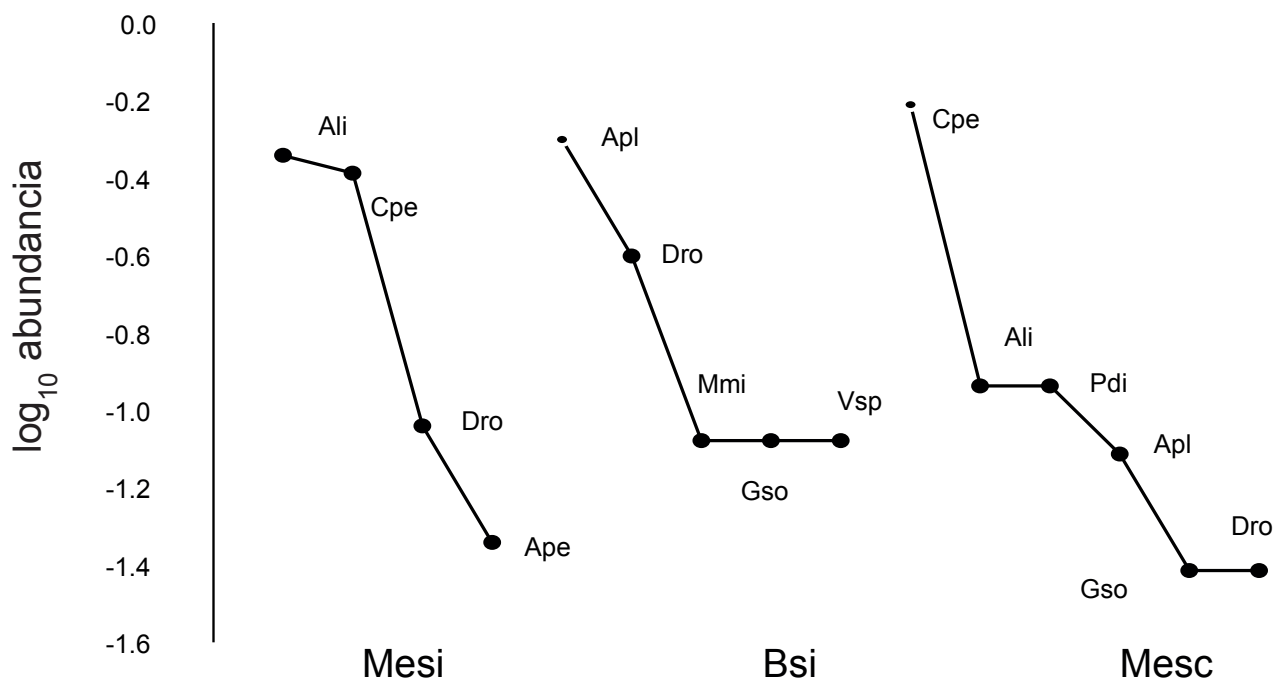

Figura 4. Curvas de rango-abundancia del ensamblaje de murciélagos en los tres sitios muestreados. Ali: A. lituratus; Ape: A. peruana; Apl: A. planirostris; Cpe: C. perspicillata; Dro: D. rotundus; Gso: G. soricina; Mmi: M. minuta; Pdi: P. discolor, Vsp: V. spectrum.
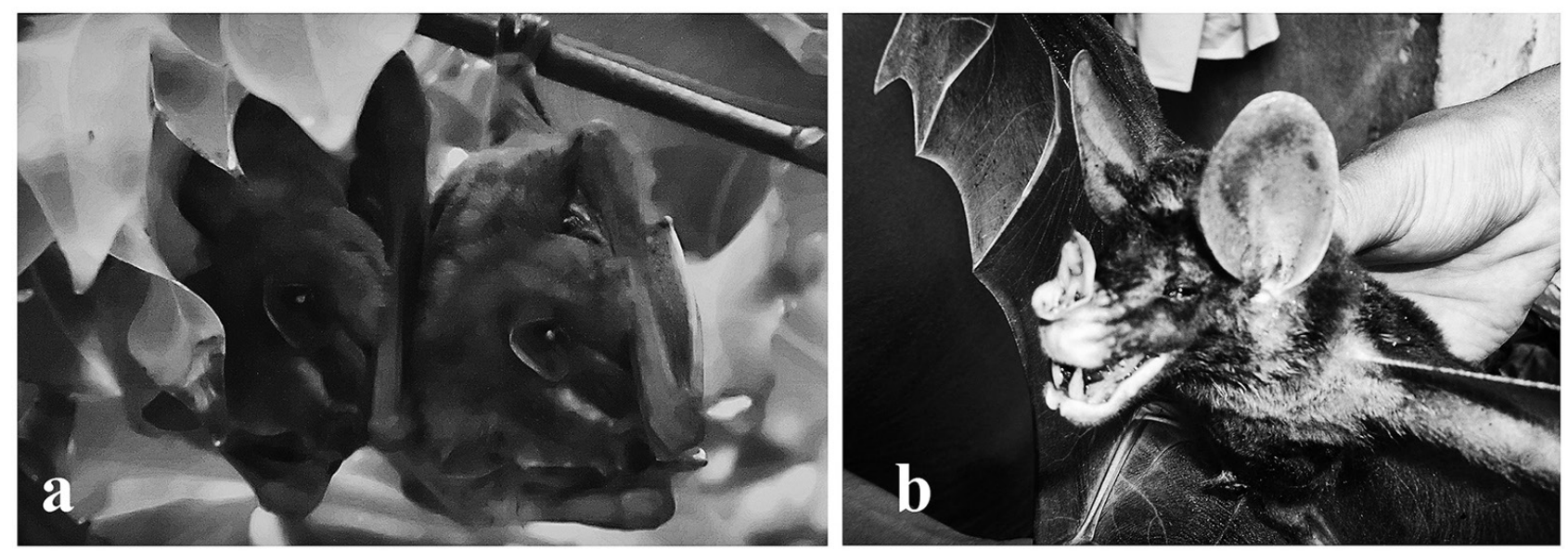

Figura 5. Murciélagos capturados durante el muestreo en el Bs-T de Nariño. a. Murciélago cara listada ( $A$. lituratus) y b. Murciélago espectral (V. spectrum) registrado en el Bosque semideciduo intervenido. 


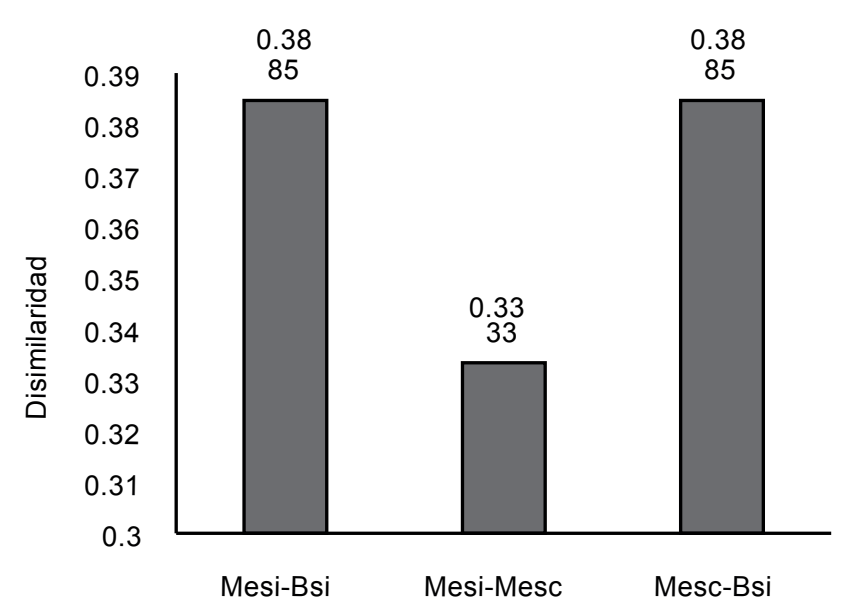

Figura 6. Diversidad beta estimada con la presencia de murciélagos entre pares de sitios. El número sobre las barras indican el valor del índice de Sorensen para cada par de sitios comparados.

Negret 1992, Sánchez et al. 2007, Ramírez-Chaves et al. 2010, Ramírez-Chaves \& Noguera-Urbano 2010) la cuenca del Patía podría tener una riqueza de 16 especies de murciélagos. Sin embargo durante el muestreo se registraron nueve especies. Como dato notable se reporta por primera vez la presencia de $G$. soricina, $M$. minuta y $V$. spectrum. Con los resultados de este estudio el nuevo número de especies para los Bs-T de Nariño y por lo tanto la cuenca del Patía en el sector Narińense sería de 19 especies.

Por otra parte se ha mencionado que el Bs-T presente en Nariño tiene muchas más afinidades históricas con ecosistemas similares del Perú y Ecuador y se encuentra separado de las zonas secas del Caribe por una barrera húmeda en Caldas y Risaralda (Colombia; Hernández-Camacho et al. 1992, Sánchez et al. 2007). Se ha propuesto que la desconexión histórica con el Caribe podría ser corroborada por la ausencia de G. longirostris y Rhogeessa io en la cuenca del Patía (Sánchez et al. 2007). Durante los muestreos realizados ninguna de las dos especies mencionadas por Sánchez et al. (2007) fueron registradas. Probablemente un mayor tiempo de muestreo y mayor cobertura espacial podría asegurar que las dos especies realmente no tienen sus distribuciones en Nariño, y con ello soportar la idea de la pertenencia de los Bs-T de Nariño al bloque del Ecuador.

La riqueza de especies de murciélagos entre los tres tipos de vegetación no tuvo diferencias, ni tampoco alcanzó el número previamente reportado por Sánchez et al. (12 especies; 2007), que hasta el momento había sido el referente sobre los murciélagos en Bs-T en Nariño. Las diferencias entre la riqueza encontrada y la reportada posiblemente se deben a que los Bs-T son ecosistemas con marcados cambios estacionales. Estos cambios influyen en la disponibilidad de recursos y por lo tanto afectan la riqueza de murciélagos entre periodos de lluvias y secas (ÁvilaCabadilla et al. 2009). Los muestreos realizados en este estudio cubrieron el inició de la época de lluvias, por lo tanto se podría esperar que la riqueza en el Bs-T de Narińo incremente al avanzar dicha época. Mayor esfuerzo de muestreo es necesario para corroborar la anterior idea. La ausencia de una o dos especies entre localidades se debe analizar con cuidado en las tres localidades considerando la baja riqueza encontrada, sin embargo las variaciones en la riqueza generalmente son asociadas con deterioro del hábitat (Jiménez-Ortega \& Mantilla-Meluk 2008).

Al considerar la riqueza de especies por familia, se ha propuesto que Phyllostomidae puede presentar mayor número de especies en los zonas con vegetación cerrada y baja perturbación (Medellín et al. 2000; Avila-Cabadilla et al. 2009, GarcíaMorales et al. 2013). Por lo tanto, el Mesc con la mayor riqueza (6 especies) posiblemente sería la zona menos perturbada de las tres analizadas. Dentro de Phyllostomidae se considera que la subfamilia Phyllostominae podría ser un indicador de buen estado de conservación, diferencias en número de especies estaría relacionado con variación en los niveles de perturbación (Medellín et al. 2000). Por lo tanto, considerando la menor riqueza de Phyllostomidae y Phyllostominae, el tipo de vegetación Mesi sería el tipo de vegetación con mayor grado de perturbación. Como se mencionó en las descripción de la zona de estudio, este tipo de vegetación es del tipo matorral y se encuentra intervenido por la presencia de cultivos (Obs. pers.). Se debe considerar que las redes de niebla permiten buena representatividad de Phyllostomidae, por lo tanto aumentar el tiempo de muestreo y hacer uso de técnicas como el monitoreo acústico o las trampas de harpa, podrían ayudar a completar el inventario y detectar mayores diferencias en la riqueza de murciélagos.

Por otra parte en el Bsi se encontraron dos especies de Phyllostominae y en el Mesc tan solo una especie de esa subfamilia. La riqueza total indica que la menor intervención la tendría el Mesc, mientras que considerando la riqueza de especies de Phyllostominae el bosque menos intervenido podría ser el Bsi. A partir de ello se podría considerar que el bajo número de especies en los dos sitios indica que los niveles de perturbación podrían ser similares.

La abundancia relativa de los murciélagos en los tres tipos de vegetación en el Bs-T de Nariño fue baja en comparación a lo encontrado en otras zonas secas del país como el cañon de Chicamocha (Obs. pers. CC-O). Esta situación ya había sido reportada con anterioridad por Sánchez et al. (2007), quienes compararon los ensamblajes de murciélagos del Patía y el Chicamocha. Los autores atribuyen las bajas abundancias a que la zona ha sufrido procesos de fragmentación y pérdida del hábitat, o al control de poblaciones realizado por personas de la zona.

Para una localidad cercana al Bsi y el Mesi denominada Remolino con vegetación de los dos tipos, se ha propuesto que es necesario un total de 42 noches de muestreo para reportar la mayoría de las especies esperadas (Sánchez et al. 2007). Lo anterior concuerda con los estimadores de riqueza analizados, que sugieren la necesidad de un mayor esfuerzo de muestreo. Por ejemplo para el Bsi el esfuerzo de muestreo permitió registrar el 75\% de la riqueza esperada de murciélagos, así que se esperarían otras dos especies con el mismo esfuerzo de muestreo. En el caso del Mesc se esperaría al menos otra especie, es decir con un mayor esfuerzo de muestreo en los dos tipos de vegetación probablemente se podría alcanzar una riqueza similar. Esto brinda limitadas evidencias que hacen suponer que los dos tipos de tipo de vegetación tienen similar estado de intervención y que las pocas diferencias en las riquezas no se deben a diferencias en el tipo de vegetación. 
La dominancia de C. perspicillata y especies de Artibeus se ha relacionado con bosques en estado de regeneración y con presencia de disturbios (Medellín et al. 2000, Rivas-Rojas 2005, Delgado-Jaramillo 2011). En el Mesc hay una mayor abundancia de la sedentaria C. perspicillata, que podría estar relacionado con la presencia de hábitat secundarios. En esa localidad se encuentra vegetación secundaria como plantas del género Piper que se recuperó de las heces de $C$. perspicillata y se detectó que frutales como Limón (Citrus sp.) y Mango (Manguifera indica) son refugio de C. perspicillata (Obs. pers.). Se ha propuesto que $C$. perspicillata es tolerante a la perturbación y puede aprovechar frutos producidos por plantas de bosques en regeneración (Rivas-Rojas 2005).

En el Mesi y el Bsi los ensamblajes de murciélagos estan dominados por especies de los murciélagos nómadas de Artibeus. Estos son murciélagos generalistas que recorren grandes distancias en busca de alimento. Su presencia en bosques de baja productividad se debe a la disponibilidad de frutos de cultivos y según Tirira (2007) a que utilizan el dosel bajo para moverse entre parches de alimentación probablemente ubicados en zonas más húmedas. En sitios con cultivos de mango, almendro, limón y mandarina se detectaron refugios de $A$. lituratus y $P$. discolor, esto puede ser un indició de la capacidad de las tres especies para soportar el deterioro y remplazamiento de su hábitat natural.

Para la especie de vampiro común (D. rotundus) se ha reportado que incrementa su abundancia en zonas altamente intervenidas (Medellín et al. 2000). En todos los sitios se registró a $D$. rotundus, sin encontrarse entre las especies más abundantes registradas durante el estudio. Sin embargo, los habitantes de la zona mencionan que los ataques a ganado son frecuentes.

Los murciélagos nectarívoros $A$. peruana y $G$. soricina presentaron abundancias bajas, lo que se puede explicar por una baja oferta de recurso floral observada en los tres sitios. En otras localidades con ecosistemas secos como el Cañon del Chicamocha y los enclaves áridos de Venezuela, se ha reportado una fuerte asociación entre cactáceas columnares y nectarívoros como vectores de polinización (Soriano \& Ruíz 2006), en este estudio no fue posible encontrar evidencia de este fenómeno.

En cuanto a diversidad beta, aunque los tres sitios presentaron especies exclusivas, el recambio no es lo suficientemente alto para considerar que son ensamblajes diferentes. Un mayor esfuerzo de muestreo es necesario para corroborar la estructura del ensamblaje de murciélagos y determinar si realmente integran uno solo.

Finalmente consideramos que los murciélagos fueron un buen indicador de la perturbación que ocurre en las tres localidades de los Bs-T de Nariño. La diferencia en la riqueza de especies, la ausencia de la subfamilia Phyllostominae en una localidad, la dominancia de especies como C. perspicillata y otras de Artibeus, y la presencia de $D$. rotundus indicaron que el Mesi (La Toma) y Bsi (El Algodonal) son las zonas con mayor grado de intervención, mientras que el Mesc (Juntas) presenta vegetación en regeneración, cultivos y es menor el grado de intervención. En la localidad de las Juntas la existencia de un área de conservación privada, podría ayudar a mantener la composición y estructura del Bs-T y servir de refugio para especies de gran movilidad como los murciélagos. Por lo tanto, el desarrollo de iniciativas como las áreas de conservación privadas cumple un importante papel en la conservación de la biodiversidad de El Algodonal y el Bs-T en Nariño.

Una de las principales causas de deterioro del Bs-T son las actividades humanas (Miles et al. 2006), por lo tanto es necesario desarrollar programas de reforestación y restauración o el fomento de sistemas agroforestales que proporcionen refugio y alimentación para las diferentes especies que habitan ese tipo de bosque. En especial los esfuerzos se deben dirigir a áreas de mayor intervención. La mayor disminución de cobertura del Bs-T ocurre en Suramérica (Miles et al. 2006). Asociar áreas de uso productivo y conservar vegetación nativa en las riveras de ríos y quebradas permitirían crear áreas de conservación de murciélagos en la zona y también de los bosques secos tropicales interandinos. En este sentido la presencia del murciélago espectral $V$. spectrum es importante, debido a que está calificado a nivel internacional como una especie casi amenazada (NT) con poblaciones aisladas y pequeñas(Aguirre et al. 2008), esta especie puede ser un objeto de conservación para la zona.

\section{Agradecimientos}

A la Asociación GAICA por las fotografías, los equipos y logística en campo. Al Museo de la Universidad de Nariño (MUN-CZ) por el ingreso para revisión de ejemplares. A The Nature Conservancy (TNC), al fondo Conserva Colombia por el apoyo económico para el desarrollo del proyecto "Planificación para la conservación de áreas en el Bs-T de Nariño”. A Fernando Burbano y Corponarińo por la gestión. A Ivan Gil de The Nature Conservancy (TNC) por el apoyo en la coordinación y desarrollo del Proyecto. EAN-U agradece al posgrado en Ciencias Biológicas de la Universidad Nacional Autónoma de México (UNAM) y al programa de becas de posgrado de CONACyT por el soporte dado durante las adecuaciones finales de este documento. A los revisores anónimos que aportaron con comentarios y sugerencias para el mejoramiento del documento.

\section{Literatura citada}

Aguirre L., H. Mantilla, B. Miller \& L. Dávalos. [2008] en línea. Vampyrum spectrum. The IUCN Red List of Threatened Species 2014.2. http://www.iucnredlist.org/ details/22843/0. Acceso 30/11/2014.

Alberíco M. \& A.J. Negret. 1992. Primer aporte sobre los mamíferos del Valle del Patía (Cauca-Nariño). Revista de Novedades Colombianas 5:66-71.

Avila-Cabadilla L.D., K.E. Stoner, M. Henry, et al. 2009. Composition, structure and diversity of phyllostomid bat assemblages in different successional stages of a tropical dry forest. Forest Ecology and Management 258(6):986-996. http://10.1016/j.foreco.2008.12.011

Chao A. \& L. Jost. 2012. Coverage-based rarefaction: standardizing samples by completeness rather than by size. Ecology 93(12):2533-2547. http://10.1890/11-1952.1

Chávez C. \& G. Ceballos. 2001. Diversidad y abundancia de murciélagos en selvas secas de estacionalidad contrastante en el Oeste de México. Revista Mexicana de Mastozoología 5:27-44.

Colwell R.K. 2013. EstimateS: statistical estimation of species richness and shared species from samples. Version 9. http://purl.oclc. org/estimates. Acceso 30/11/2014.

Delgado-Jaramillo M., M.J. Machado F. García, \& J. Ochoa. 2011. Murciélagos (Chiroptera: Mammalia) del Parque Nacional Yurubí, Venezuela: listado taxonómico y estudio comunitario. Revista de Biología Tropical 59(4):1757-1776.

Delgado A., S. Ruiz, L. Arévalo, et al. 2007. Plan de Acción en Biodiversidad del departamento de Nariño 2006 - 2030 - Propuesta Técnica. Corporación Autónoma Regional de Nariño - Corponariño, Gobernación de Nariño - Secretaría 
de Agricultura, Instituto de Investigación de Recursos Biológicos Alexander von Humboldt - IAvH, Universidad de Nariño, Unidad Administrativa Especial del Sistema de Parques Nacionales Naturales - UAESPNN, Universidad Mariana, Asociación para el Desarrollo Campesino - ADC, Asociación de Consejos Comunitarios y Organizaciones Étnico Territoriales de las zonas Centro y Norte de la Costa Pacífica de Nariño - ASOCOETNAR y Red de Consejos Comunitarios del Pacífico Sur - RECOMPAS. Pasto, 196 p. <http://corponarino.gov.co/expedientes/intervencion/ biodiversidad/parteI.pdf. Acceso: 15/12/2013.

García H., G. Corzo, P. Isaacs \& A. Etter. 2014. Distribución y estado actual de los remanentes del bioma de Bosque Seco Tropical en Colombia: insumos para su gestión. En: El Bosque Seco Tropical en Colombia. C. Pizano \& H. García. Instituto de Investigación de Recursos Biológicos Alexander von Humboltd (IAvH). Bogotá. Pp. 229-251.

García-Morales R., E.I. Badano \& C.E Moreno. 2013. Response of neotropical bat assemblages to human land use. Conservation Biology 27(5): 1096-1106. http:// 10.1111/ cobi.12099.

Gotelli N.J. \& R.K. Colwell. 2011. Estimating species richness. En: Frontiers in measuring biodiversity. A.E. Magurran \& B.J. McGill (eds). Oxford University. New York. Pp. 39-54.

Hernández-Camacho J.I., T. Walshburger, R. Ortiz- Quijano, et al. 1992. Origen y Distribución de la Biota Suramericana y Colombiana. En: La diversidad biológica de Iberoamérica I. Halffter G. C. (ed.). Xalapa, México: Instituto de Ecología. Pp. 55-104.

Hsieh, T.C., K.H. Ma \& A. Chao. 2015. iNEXT: An interpolation and extrapolation method of species diversity (Hill numbers). http://chao.stat.nthu.edu.tw/blog/software-download/. Acceso 30/11/2015.

Instituto Alexander von Humboldt (IAVH). 1998. El Bosque Seco Tropical (bs-T) en Colombia. IAVH, Villa de Leyva, Colombia. http://media.utp.edu.co/ciebreg/archivos/bosqueseco-tropical/el-bosque-seco-tropical-en-colombia.pdf. Acceso 30/11/2014

Jiménez-Ortega A. \& H. Mantilla-Meluk. 2008. El papel de la tala selectiva en la conservación de bosques neotropicales y la utilidad de los murciélagos como bioindicadores de disturbio. Revista Institucional Universidad Tecnológica del Chocó: investigación biodiversidad y desarrollo 27(1):100-108.

Jost L. 2006. Entropy and diversity. Oikos 113(2): 363-375. http:// $10.1111 / \mathrm{j} .2006 .0030-1299.14714 . \mathrm{x}$
Jurado R.D. \& E. Herrera. 2014. Caracterización florística del bosque seco narińense municipios de El Peñol y Taminango - Colombia. Informe Técnico. Asociación GAICA. Pp.9-12.

Mantílla-Meluk H. \& R.J. Baker. 2010. New species of Anoura (Chiroptera: Phyllotosmidae) from Colombia, with systematic remarks and notes on the distribution of the A. geoffroyi complex. Occasional Papers Museum of Texas Tech University 292:1-19. http://www.nsrl.ttu.edu/publications/ opapers/ops/OP292.pdf.

Medellín R.A., M. Equihua \& M.A. Amin. 2000. Bat diversity and abundance as indicators of disturbance in Neotropical rainforests. Conservation Biology 14(6):1666-1675. http:// 10.1111/j.1523-1739.2000.99068.x

Miles L., A.C. Newton, R.S DeFries, et al. 2006. A global overview of the conservation status of tropical dry forests. Journal of Biogeography 33(3):491-505. http:// 10.1111/j.15231739.2000.99068.x.

Moreno C.E. 2001. Métodos para medir la biodiversidad. M\&TManuales y Tesis SEA, vol.1. Zaragoza. Pp.84.

Ramírez-Chaves H., F. Ayerbe-Quiñones \& O. Mejía-Egas. 2010. Mamíferos de la cuenca alta del río Patía en el departamento del Cauca, Colombia. Boletín Científico, Centro de Museos, Museo de Historia Natural 14:92-113.

Ramírez-Chávez H. \& E.A. Noguera-Urbano. 2010. Lista preliminar de los mamíferos (Mammalia: Theria) del departamento de Narińo, Colombia. Biota Colombiana 11 (1 y 2):117-140.

Rivas-Rojas E. 2005. Diversity bats of dry forest and cocoa plantation. Lyona a journal of ecology and application 8(2):29-39.

Ruíz A., M.Santos, P.J. Soriano, J. Cavelier \& A. Cadena. 1997. Relaciones mutualísticas entre el Murciélago Glossophaga longirostris y las cactáceas columnares en la zona árida de La Tatacoa, Colombia. Biotropica 29(4):469-479.

Sánchez F., J. Alvarez, C. Ariza \& A. Cadena. 2007. Bat assemblage structure in two dry forests of Colombia: Composition, species richness, and relative abundance. Mammalian Biology 72 (2):82-92. http:// 10.1016/j.mambio.2006.08.003

Simmons N.B. \& R.S. Voss. 2007. Collection, preparation, and fixation of specimens and tissues. In: Ecological and behavioral methods for the study of bats, 2nd Edition (T. H. Kunz and S. Parsons, eds.). Johns Hopkins University Press. Pp. 849-867

Tirira D. 1998. Historia Natural de los murciélagos Neotrópicales. En Biología, Sistemática y Conservación de los mamíferos del Ecuador. Museo de Zoología, Centro de Biodiversidad y Ambiente. Pontificia Universidad Católica del Ecuador. Publicación especial 1. Pp. 31-56. 